\title{
Descrição da larva de Popanomyia femoralis Kertész, 1909 e do pupário de Engicerus major Lindner, 1964 (Diptera, Stratiomyidae)
}

\author{
Roberto de Xerez ${ }^{1}$ \\ José Roberto Pujol-Luz ${ }^{2}$ \\ Gustavo Glória Viana ${ }^{1,3}$
}

\begin{abstract}
Aвstract. Description of the larva of Popanomyia femoralis Kertész, 1909 and puparium of Engicerus major Lindner, 1964 (Diptera, Stratiomyidae). The larva of Popanomyia femoralis and puparium of Engicerus major are described. The larvae were collected under the bark of fallen trees in an area near the tropical rain forest at Ilha da Marambaia

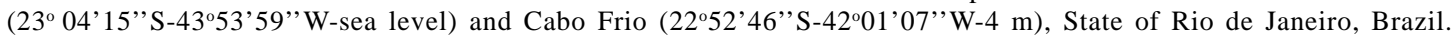
Biological notes are presented. The larva of Popanomyia femoralis and puparium of Engicerus major are compared with those of Chalcidomorphina aurata Enderlein, Cosmariomyia argyrosticta Kertész, Dactylodeictes lopesi Lindner and Vittiger schnusei Kertész, other species of the same subfamily.
\end{abstract}

Keywords. Engicerus major; Pachygastrinae; Popanomyia femoralis; soldier flies; Stratiomyidae.

\section{INTRODUÇÃO}

Das 136 espécies de Pachygastrinae ocorrentes na região Neotropical (Woodley 2001), são conhecidas e descritas as larvas e/ou pupários de somente seis espécies: Eupachygaster alexanderi (James, 1967), Zabrachia stoichoides James, 1965, Chalcidomorphina aurata Enderlein, 1914, Vittiger schnusei Kertész, 1909, Cosmariomyia argyrosticta Kertész, 1914 e Dactylodeictes lopesi Lindner, 1964 (BlanchaRd 1923; JAMES 1965; Pujol-Luz \& Xerez 1999; Xerez \& Pujol-Luz 2001; Xerez et al. 2002, respectivamente).

Popanomyia Kertész, 1909, com duas espécies, distribuise na região Neotropical pelo Panamá, Brasil e Peru: P. femoralis Kertész, 1909 (Peru, Brasil - Rio de Janeiro e Santa Catarina) e $P$. kerteszi James \& Woodley, 1980 (Panamá e Peru). Engicerus Lindner, 1964, com uma espécie, é encontrada na região Neotropical somente no Brasil: E. major Lindner, 1964 (Brasil Rio de Janeiro e Santa Catarina) (Woodley, 2001).

Neste trabalho, descrevemos a larva e o pupário de Popanomyia femoralis e o pupário de Engicerus major e os comparamos com as larvas e/ou pupários de C. aurata, C. argyrosticta, D. lopesi e V. schnusei.
As larvas de $P$. femoralis foram coletadas na Ilha da

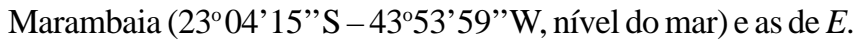
major em Cabo Frio (22 52' 46”'S - 42 01'07'”W, 4 m), Rio de Janeiro, Brasil, em ambos os casos sob a casca de árvores caídas e em estágio inicial de decomposição. Foram coletadas 80 larvas de $P$. femoralis e uma de E. major.

Toda a metodologia de criação segue Pujol-Luz \& Xerez (1999), Xerez \& Pujol-Luz (2001) e Xerez et al. (2002).

O material coletado foi depositado na Coleção Entomológica Costa Lima, Universidade Federal Rural do Rio de Janeiro, Seropédica, RJ, Brasil.

\section{Popanomyia femoralis Kertész, 1909}

(Figs. 1-5)

Distribuição. Panamá, Peru e Brasil: Rio de Janeiro (nova ocorrência)

$$
\text { e Santa Catarina. }
$$

Larva (e pupário). Comprimento 6,0 a 8,2 mm, achatada dorsoventralmente, margens laterais dos segmentos do corpo fortemente arqueadas. Cutícula com a aparência usual de mosaico. Padrão cromático marrom-amarelado.

\footnotetext{
1. Departamento de Biologia Animal, Instituto de Biologia, Universidade Federal Rural do Rio de Janeiro. Rodovia BR- 465 km 07, 23890-000 Seropédica-RJ, Brasil. Endereço eletrônico: rdexerez@ufrrj.br

2. Departamento de Zoologia, Instituto de Biologia, Universidade de Brasília. 70910-900 Brasília-DF. Endereço eletrônico: jrpujol@unb.br

3. Graduando em Ciências Biológicas. Endereço eletrônico: ggviana@ufrrj.br
} 
Cabeça. Achatada dorsoventralmente; comprimento maior do que a largura (Figs. 1, 2); complexo mandíbulo-maxilar bem desenvolvido, escovas cilíndricas quase tão longas quanto o labro em vista dorsal; labro triangular. Antenas pequenas, surgindo na parte anterior da cabeça. Olhos proeminentes, arredondados, situados na parte posterior da cabeça. Quetotaxia: 2 pares de cerdas clipeofrontais; 1 par de cerdas dorsolaterais inseridas acima dos olhos; 1 par de cerdas laterais inseridas abaixo dos olhos (Figs. 1, 2); 3 pares de cerdas ventrolaterais e 3 pares de cerdas ventrais em forma de espinho (Fig. 2).

Tórax. Primeiro segmento torácico menor que os outros; espiráculo com 2 cerdas curtas à frente, 1 dorsolateral, 1 ventrolateral; com duas fileiras de cerdas: 2 pares de cerdas anterodorsais, 3 pares de cerdas dorsais, sendo o primeiro par situado atrás da linha das cerdas anterodorsais (Fig. 1) e 2 pares de cerdas ventrais, sendo o par mais externo bifurcado (Fig. 2). Segundo e terceiro segmentos com uma fileira de cerdas com 3 pares de cerdas dorsais, 2 pares de cerdas ventrais, sendo o par mais externo bifurcado, 1 par de cerdas laterais e 1 par de cerdas ventrolaterais (Figs. 1, 2).

Abdômen. Primeiro ao sétimo segmentos com a mesma forma, uma fileira de 3 pares de cerdas dorsais sendo o mais externo mais curto do que os outros (Fig. 3); 3 pares de cerdas ventrais sendo o par mais externo mais curto do que os outros (Fig. 4), 4 pares de cerdas laterais abdominais (1 par dorsolateral - 1 par lateral - 2 pares ventrolaterais) (Fig. 5); linha ventromediana do sexto segmento com uma mancha característica elíptica uniforme (Fig. 4); oitavo segmento arredondado, com 1 par de cerdas dorsocentrais (Fig. 3), 5 pares de cerdas ventrais; 2 pares de cerdas laterais, 1 par subapical e 1 par apical, todos do mesmo tamanho (Figs. 3, 4).

O pupário apresenta as mesmas características da larva de último instar.

Material examinado. BRASIL. Rio de Janeiro: Ilha da Marambaia, 08.IV.1998 e 15.IV.1998, R. de Xerez col., 11 pupários; 42 exemplares (emergências: 30.VII.1998, 01-03.VIII.1998, 04.VIII.1998, 05.VIII.1998, 08-10.VIII.1998, 14.VIII.1998, 20.VIII.1998, 2224.VIII.1998, 02.IX.1998, 04.IX.1998, 17.IX.1998 e 17.X.1998; 0103.VIII.1998, 04.VIII.1998 e 05.VIII.1998), 6 larvas (último ínstar).

Engicerus major Lindner, 1964

(Figs. 6-10)

Distribuição. Brasil: Rio de Janeiro (nova ocorrência) e Santa Catarina.

Pupário. Comprimento 5,9 mm, achatado dorsoventralmente, margens laterais dos segmentos do corpo fortemente arqueadas. Cutícula com a aparência usual de mosaico, algumas células formando manchas características e placas no sexto e oitavo segmentos abdominais. Padrão cromático marrom-amarelado.

Cabeça. Achatada dorsoventralmente; comprimento maior do que a largura (Figs. 6, 7); complexo mandíbulo-maxilar bem desenvolvido, escovas cilíndricas quase tão longas quanto o labro em vista dorsal; labro triangular. Antenas curtas, surgindo na parte anterior da cabeça. Olhos proeminentes, arredondados, situados na parte posterior da cabeça. Quetotaxia: 2 pares de cerdas clipeofrontais; 1 par de cerdas dorsolaterais inseridas acima dos olhos e 1 par de cerdas laterais inseridas abaixo dos olhos (Figs. 6, 7); 3 pares de cerdas ventrolaterais e 3 pares de cerdas ventrais em forma de espinho (Fig. 7).

Tórax. Primeiro segmento torácico menor que os outros; espiráculo proeminente, em vista lateral circundado por 1 par de cerdas dorsolaterais e 1 par de cerdas ventrolaterais; com 2 fileiras de cerdas: 2 pares de cerdas anterodorsais, sendo o par mais interno menor, 3 pares de cerdas dorsais, sendo o primeiro par aproximadamente na mesma linha que as anterodorsais (Fig. 6) e 2 pares de cerdas ventrais sendo o par mais externo bifurcado (Fig. 7). Segundo e terceiro segmentos com uma fileira de cerdas com 3 pares de cerdas dorsais, 2 pares de cerdas ventrais sendo o mais externo bifurcado, 1 par de cerdas laterais e 1 par de cerdas ventrolaterais.

Abdômen. Primeiro ao sétimo segmentos com a mesma forma, uma fileira de 3 pares de cerdas dorsais com tamanho decrescente do par mais interno para o mais externo (Fig. 8); 3 pares de cerdas ventrais sendo o par mediano mais curto do que os outros (Fig. 9); 4 pares de cerdas laterais abdominais (1 par dorsolateral - 1 par lateral -2 pares ventrolaterais) (Fig. 10); linha ventromedial do sexto segmento com uma mancha característica elíptica alongada com um ligeiro estreitamento central (Fig. 9). Oitavo segmento com 1 par de cerdas dorsocentrais (Fig. 8), 5 pares de cerdas ventrais, 2 pares de cerdas laterais, 1 par apical e 1 par subapical menor do que os outros.

Material examinado. BRASIL. Rio de Janeiro: Búzios, Estação Rádio da Marinha em Campos Novos, 06.II.1999 R. de Xerez col., 1 pupário, 1 fêmea (emergência: 11.III.1999).

\section{DISCUSSÃO}

Popanomyia femoralis pode ser identificada pela seguinte combinação de caracteres: primeiro par de cerdas dorsais do primeiro segmento torácico situado atrás da linha das cerdas anterodorsais; par mais externo das cerdas dorsais abdominais mais curto do que os outros dois pares (mediano e mais interno); cerdas ventrais abdominais aproximadamente do mesmo tamanho; mancha esternal do sexto segmento abdominal elíptica, uniforme. Engicerus major difere de $P$. femoralis por apresentar o primeiro par de cerdas dorsais do primeiro segmento torácico aproximadamente na mesma linha das cerdas anterodorsais; as cerdas dorsais abdominais apresentam-se em tamanho decrescente do par mais interno para o mais externo; o par mediano das cerdas ventrais abdominais é menor do que os pares interno e externo; mancha esternal do sexto segmento abdominal, elíptica com um estreitamento na região central.

Também foi observado nestas duas espécies, assim como em pupários das quatro outras espécies estudadas pelos autores (Chalcidomorphina aurata , Cosmariomyia argyrosticta, Dactylodeictes lopesi e Vittiger schnusei), o aparecimento, próximo à emergência, de uma linha transversal entre o primeiro e o segundo segmentos torácicos e de uma linha dorso-mediana a partir do segundo segmento torácico 

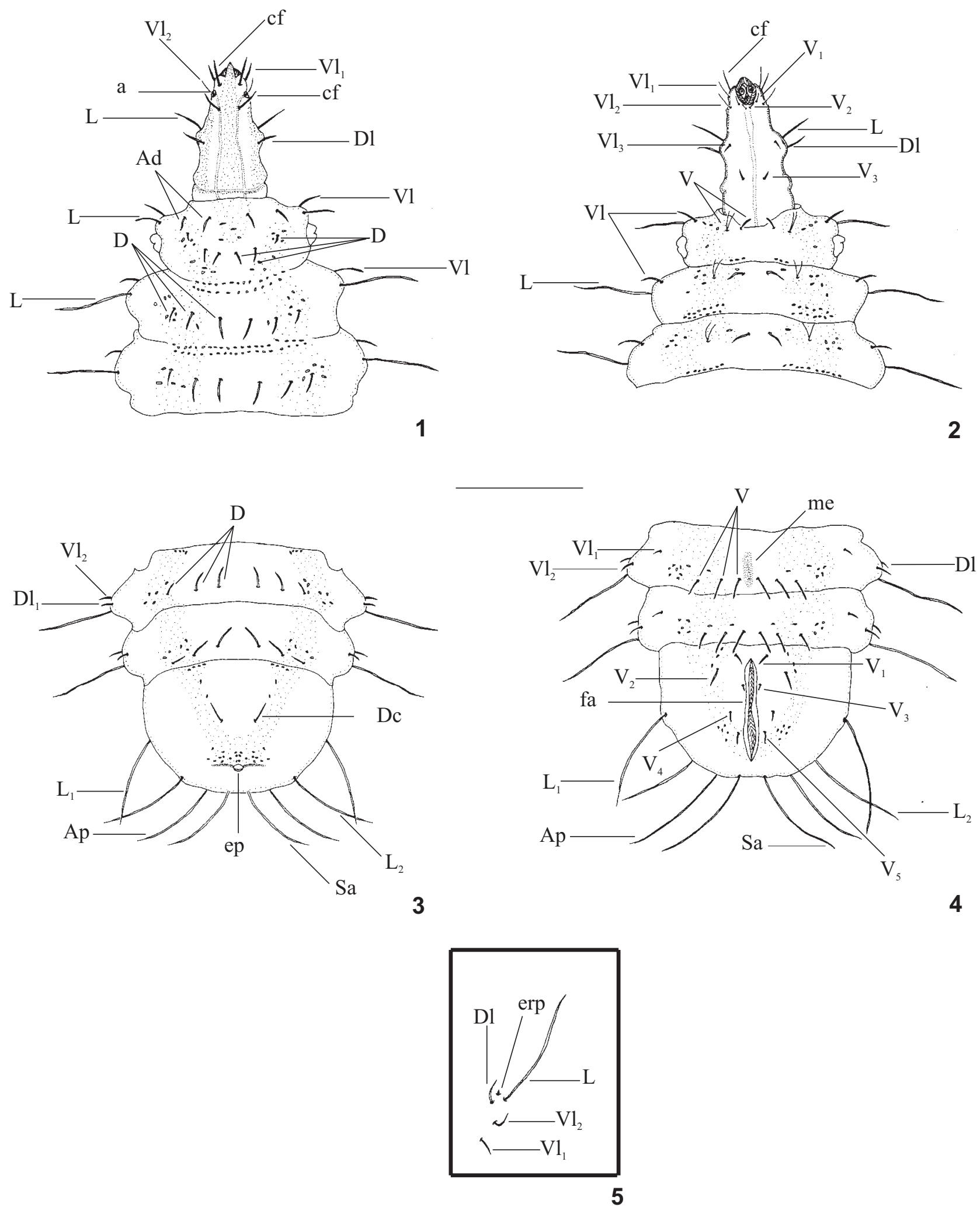

Figs. 1-5. Popanomyia femoralis Kertész. 1, cabeça e $1^{\circ}, 2^{\circ}$ e $3^{\circ}$ segmentos torácicos (vista dorsal); 2, cabeça e $1^{\circ}$, $2^{\circ}$ e $3^{\circ}$ segmentos torácicos (vista ventral); 3, $6^{\circ}, 7^{\circ}$ e $8^{\circ}$ segmentos abdominais (vista dorsal); 4, $6^{\circ}, 7^{\circ}$ e $8^{\circ}$ segmentos abdominais (vista ventral); 5 , esquema das cerdas da região lateral dos segmentos abdominais; $\mathrm{a}=$ antena; $\mathrm{Ad}=$ cerdas anterodorsais; $\mathrm{Ap}=$ cerdas apicais; $\mathrm{cf}=$ cerdas clipeofrontais; $\mathrm{D}=$ cerdas dorsais; Dc $=$ cerdas dorsocentrais; $\mathrm{Dl}=$ cerdas dorsolaterais; ep = espiráculo posterior; erp $=$ espiráculo respiratório pupal; fa $=$ fenda anal; $\mathrm{L}=$ cerdas laterais; me $=$ mancha esternal; $\mathrm{Sa}=$ cerdas subapicais; $\mathrm{V}=$ cerdas ventrais; $\mathrm{Vl}=$ cerdas ventrolaterais. Barra $=1 \mathrm{~mm}$. 

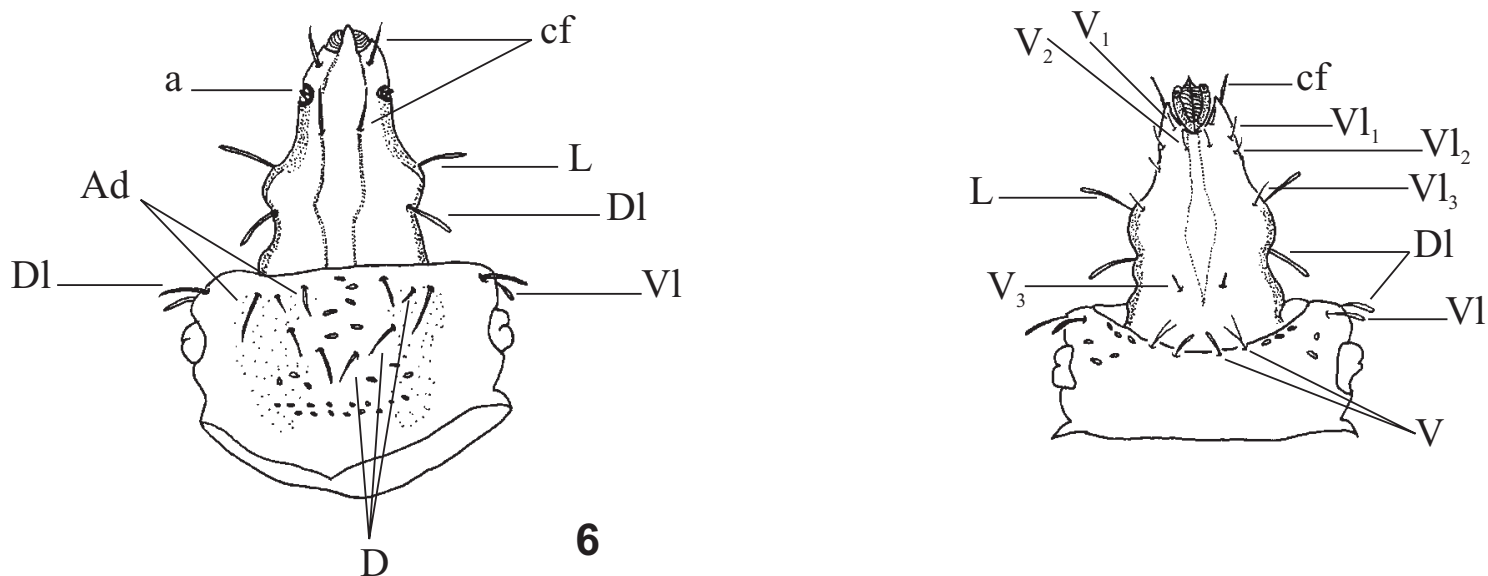

$\mathrm{D}$
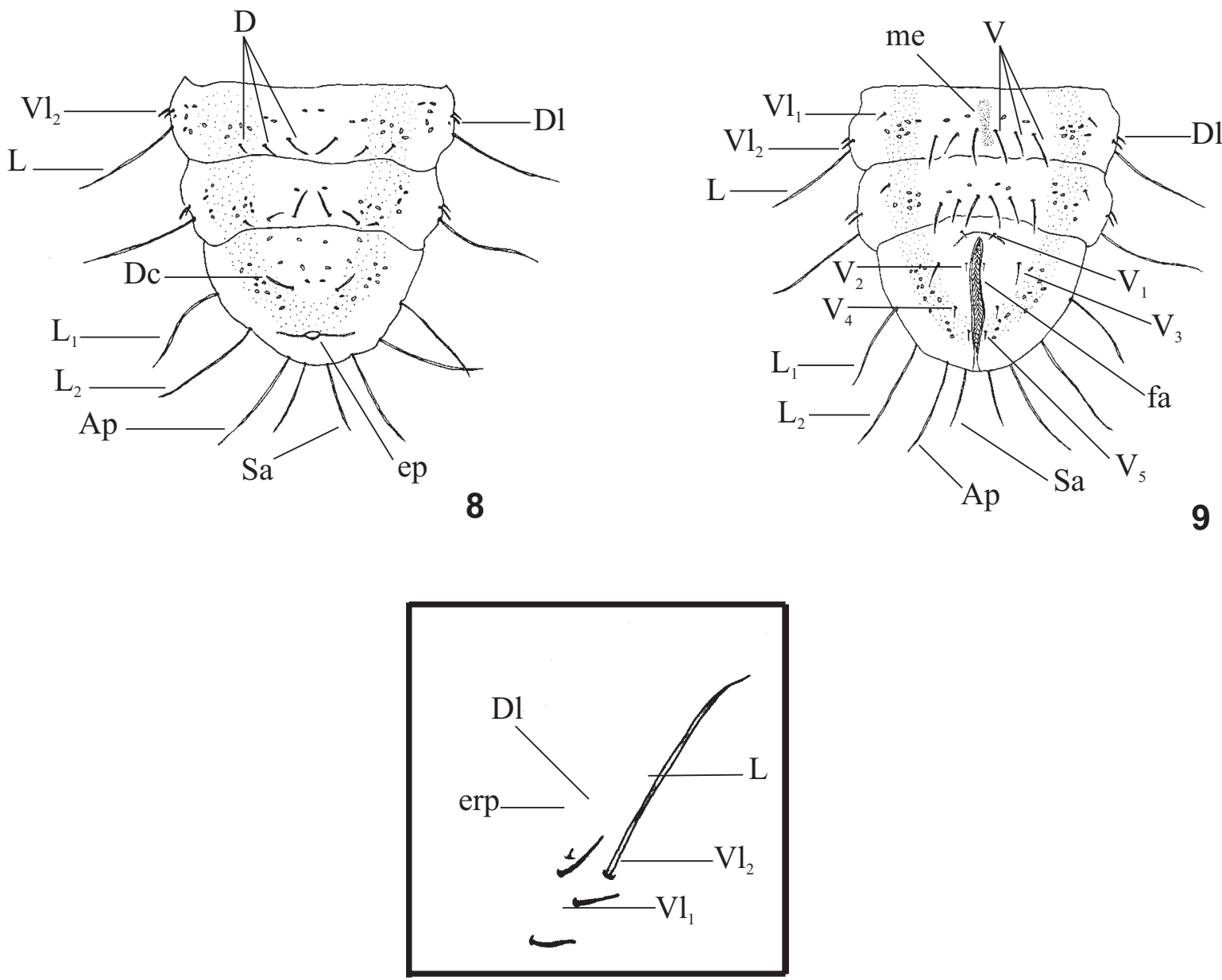

10

Figs. 6-10. Engicerus major Lindner. 6, cabeça e $1^{\circ}$ segmento torácico (vista dorsal); 7, cabeça e $1^{\circ}$ segmento torácico (vista ventral); 8, $6^{\circ}, 7^{\circ}$ e $8^{\circ}$ segmentos abdominais (vista dorsal); 9, $6^{\circ}, 7^{\circ}$ e $8^{\circ}$ segmentos abdominais (vista ventral); 10, esquema das cerdas da região lateral dos segmentos abdominais; a = antena; Ad = cerdas anterodorsais; $\mathrm{Ap}=$ cerdas apicais; $\mathrm{cf}=$ cerdas clipeofrontais; $\mathrm{D}=$ cerdas dorsais; $\mathrm{Dc}=$ cerdas dorsocentrais; $\mathrm{Dl}$ = cerdas dorsolaterais; ep $=$ espiráculo posterior; erp $=$ espiráculo respiratório pupal; fa $=$ fenda anal; $\mathrm{L}=$ cerdas laterais; me $=$ mancha esternal; $\mathrm{Sa}=$ cerdas subapicais; $\mathrm{V}=$ cerdas ventrais; $\mathrm{Vl}=$ cerdas ventrolaterais. Barra $=1 \mathrm{~mm}$. 
atingindo até o terceiro segmento torácico e, às vezes, até o primeiro segmento abdominal.

RozKošnÝ \& Kovac (1991) descreveram o pupário de Camptopteromyia fractipennis Meijere, 1914 (Pachygastrinae - região Oriental) e citaram a presença de um pequeno espiráculo respiratório pupal em forma de bastão nas laterais do primeiro ao quinto segmentos abdominais, próximo à cerda lateral. Os mesmos autores (RozKoŠNÝ \& KovAC 1998a), ao descreverem o pupário de Pachygaster piriventris Rozkošný \& Kovac 1998 (Pachygastrinae - região Oriental), citaram a presença de espiráculos, com a mesma forma, numa posição dorsolateral entre as cerdas laterais. Ainda, os mesmos autores (RozkošNÝ \& Kovac 1998b) comentaram que o período pupal pode ser detectado por algumas características, dentre as quais a presença de espiráculos respiratórios pupais inconspícuos, pequenos e em forma de bastão, presentes na região dorsolateral do primeiro ao sexto segmentos abdominais (Beridinae e Pachygastrinae) ou do segundo ao quinto segmentos abdominais (Clitellariinae). As larvas de P. femoralis e E. major também apresentam espiráculos numa posição dorsolateral do primeiro ao sexto segmentos abdominais, formando um pequeno triângulo com as inserções das cerdas (Figs. 5, 10), característica também observada em C. aurata, C. argyrosticta e V. schnusei. Nesta última, os espiráculos abdominais estão presentes do primeiro ao sétimo segmentos. Em $D$. lopesi, os espiráculos estão presentes do primeiro ao sexto segmentos abdominais, porém, formando uma linha reta com a inserção das cerdas.

Um outro aspecto observado em $P$. femoralis e em $C$. argyrosticta, foi o desenvolvimento de espiráculos respiratórios pupais, pois, com o grande número de larvas coletadas, foi possível observar que os espiráculos se tornavam mais visíveis à medida que ocorria o desenvolvimento da pupa, acreditando-se, desta forma, que o seu desenvolvimento seja gradual.

TeSkey (1976) afirmou que as larvas de Stratiomyidae são normalmente gregárias. Este fato foi verificado na maioria de nossas coletas, excetuando-se $D$. lopesi e E. major, das quais só foi coletado um exemplar de cada uma das espécies. Uma observação importante é o fato de que, na época das duas coletas realizadas, os troncos nos quais as larvas foram coletadas estavam expostos ao sol, secos e parcialmente descascados, o que pode ter ocasionado a morte das demais larvas.

Vários autores (e.g. KRAFT \& CoOK 1961; McFAdDEN 1967; JAMES 1967; KRIVOSHeINA 1977; RozKošNÝ \& KovaC 1998a ; Pujol-Luz \& Xerez 1999; Xerez \& Pujol-Luz 2001; Xerez et al. 2002) citaram que, normalmente, as larvas de Pachygastrinae estão associadas a árvores ou galhos de árvores caídos e em decomposição, onde são encontradas sob a casca. O grau de decomposição da árvore pode influenciar na presença ou não de larvas. Em nossas coletas, quando procurávamos sob a casca de árvores recém-caídas ou num adiantado estado de decomposição, não encontrávamos nenhuma larva. Acreditamos que este fato pode estar relacionado com o seu hábito alimentar que, segundo Кraft \& Соок (1961), McFadden (1967), Teskey (1976), é constituído de seiva fermentada, esporos de fungos ou microorganismos produtores de decomposição, provavelmente bactérias. As larvas de $P$. femoralis foram coletadas em troncos que apresentavam um certo grau de umidade e uma grande quantidade de fungos e restos de material em decomposição, o que corrobora a afirmativa dos autores supracitados. A larva de E. major foi coletada num tronco parcialmente descascado e exposto ao sol, o que explicaria a única larva presente. Em troncos com formigas e/ou cupins não foram encontradas larvas.

Agradecimentos. Ao Centro de Adestramento da Ilha da Marambaia (CADIM - Marinha do Brasil), na pessoa de seu Comandante, na época da coleta, Capitão de Mar-e-Guerra (FN) Mário Sérgio Pacheco de Souza; ao Imediato do CADIM, Capitão-de-Fragata (FN) Djair da Silva Azevedo, pelo contato com o Comandante da Estação Rádio da Marinha em Campos Novos, Cabo Frio, RJ; ao Comandante da Estação Rádio da Marinha em Campos Novos, Capitão de Fragata Marcelo Augusto da Cunha Porto, pela possibilidade de coleta na área da OM. Ao Curso de Pós-Graduação em Biologia Animal (UFRRJ) pelas facilidades oferecidas durante a realização da minha tese de Doutorado (RdX). Ao Conselho Nacional de Desenvolvimento Científico e Tecnológico - CNPq pelo auxílio processo n ${ }^{\circ}$ 300265/96-4 (JRPL) e ao Programa Institucional de Bolsas de Iniciação Científica da Universidade Federal Rural do Rio de Janeiro (GGV).

\section{REFERENNCIAS}

Blanchard, E. E. 1923. Apuntes sobre dos dípteros argentinos. Physis 6: 319-323.

JAMES, M. T. 1965. Contributions to our knowledge of the nearctic Pachygasterinae (Diptera: Stratiomyidae). Annals of the Entomological Society of America 58: 902-908.

JAmES, M. T. 1967. Bredin-Archbold-Smithsonian Biological Survey of Dominica. 5. Family Stratiomyidae (Diptera). Proceedings of the United States National Museum 123(3622): 1-22.

Kraft, K. J. \& E. F. Соок.1961. A revision of the Pachygasterinae (Diptera, Stratiomyidae) of America North of Mexico. Miscellaneus Publications of the Entomological Society of America 3(1): 1-24.

KRIVosheina, N. P. 1977. Xylophilous larvae of soldier flies of the subfamily Pachygasterinae (Diptera, Stratiomyidae). Entomological Review 56(4): 113-122.

McFadDEN, M. W. 1967. Soldier fly larvae in America North of Mexico. Proceedings of the United States National Museum 121(3569): $1-72$.

Pujol-Luz, J. R. \& R. De Xerez. 1999. The larva of Chalcidomorphina aurata Enderlein, 1914 (Diptera: Stratiomyidae) from "Ilha da Marambaia”, Rio de Janeiro, Brazil. Proceedings of the Entomological Society of Washington 101: 295-299.

RozкоšnÝ, R. \& D. KovaK. 1991. First description of the male and the larva of Camptopteromyia fractipennisde de Meijere from Malaysia (Diptera: Stratiomyidae). Entomologica Scandinavica 22(3): 297304.

RozkošnÝ, R. \& D. KovaK. 1998a. A new species of Pachygaster (Diptera: Stratiomyidae, Pachygasterinae), from West Malaysia and Thailand. Studia Dipterologica 5(1): 3-12.

RozKošNÝ, R. \& D. KоVAK. 1998b. Descriptions of bamboo-inhabiting larvae and puparia of Oriental soldier flies Ptecticus brunettii and $P$. flavifemoratus (Diptera: Stratiomyidae: Sarginae) with observations on their biology. European Journal of Entomology 95: 65-86.

TeSKey, H. J. 1976. Diptera larvae associated with trees in North America. Memoirs of the Entomological Society of Canada 100: 1-53. Woodley, N. E. 2001. A world catalog of the Stratiomyidae (Insecta: Diptera). Myia 11: 1-473.

Xerez, R. DE \& J. R. Pujol-Luz. 2001. Description of the larva of Vittiger 
schnusei Kertész, 1909 (Diptera, Stratiomyidae) from Ilha da Marambaia, Rio de Janeiro, Brasil. Studia Dipterologica 8(1): 337-341.

Xerez, R. de; J. R. Pujol-Luz \& G. G. Viana. 2002. Descrição da larva de Cosmariomyia argyrosticta Kertész, 1914 e do pupário de Dactylodeictes lopesi Lindner, 1964 (Diptera, Stratiomyidae).

Revista Brasileira de Zoologia 19(3): 747-755. 